\title{
On the value of the mindsponge* bayesvl approach to researching humanities and social sciences phenomena
}

Minh-Hoang Nguyen \& Manh-Toan Ho

\author{
AISDL \\ Vuong \& Associates \\ Dong Da, Hanoi 100000, Vietnam
}

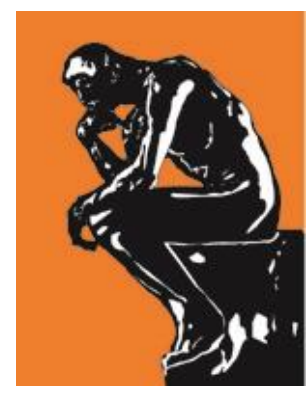

Centre for Interdisciplinary Social Research

Phenikaa University

Yen Nghia, Ha Dong, Hanoi 100803, Vietnam

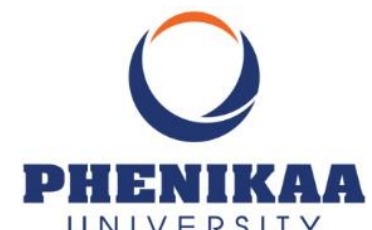

UNIVERSITY

May 23, 2021

Since the three most important methodological discoveries by AISDL (Vuong \& Associates) and the Centre for Interdisciplinary Social Research (ISR) [1], Phenikaa University, our team members and we have been striving to apply these findings in scientific research. Some of the initial efforts are fruitful, while later endeavors are waiting to be cultivated.

In this discussion, we mark the ISR's first milestone of applying the mindsponge mechanism $[2,3]$ together with the bayesvl package $[4,5,6]$ by summarizing the current progress. Moreover, we 
also present how to reapply the mindsponge*bayesvl approach in future studies, which can be a helpful guideline for both ISR team members and other scholars.

Three significant findings in multiple disciplines have been discovered using the mindsponge mechanism and the bayesvl package. First, we offered a new information-processing approach to study the formulation mechanism of suicidal ideation in an individual's mind [7]. Second, we proposed a psycho-religious mechanism to explain the psychology of suicide attackers using a trustbased evaluation mechanism and cost-benefit judgment among religious individuals [8]. Third, the information-processing approach was also expanded to the discipline of educational psychology by exploring the pathways to book reading interest among secondary school students [9]. Besides these significant findings, the mindsponge mechanism and bayesvl package have also shed light on several socio-cultural phenomena, such publications as [10,11,12,13]

The theoretical backbones of these three findings are constructed based on the mindsponge mechanism. At the same time, their justifications are aided by the logical construction and flexibility of Bayesian inference provided by the bayesvl package. Such methodology is also applied in upcoming projects of the Centre ISR. Here, we outline important stages of conducting research using the mindsponge mechanism and bayesvl package for transparently disseminating the knowledge related to the methodology [14].

First of all, it is necessary for the user to acquire knowledge of the mindsponge mechanism's components and structure. Such information can be found here [2,3].

Next, the user needs to determine the subject or problem that they want to investigate. As the information exchanging process between the individual's mindset and the environment is a fundamental component of the mindsponge mechanism, understanding the studied problem's contexts is required (e.g., environmental, economic, and socio-cultural contexts).

After determining the research problem and its context, the user needs to identify the factors of the problems and their proxies. During this process, the user should be greatly conscious of the feasibility of employing available data for choosing studied factors for the sake of cost-saving [15].

The mindsponge mechanism should be interpreted as a framework to construct logical connections between factors of the studied problem. Thus, after gathering enough factors to build a model, the user can build a logical network based on the reasoning of the mindsponge framework.

The bayesvl package offers Bayesian inference and supports constructing a logical network before model fitting [16,17]. The user can be highly flexible in constructing a logical network due to the advantages of Bayesian inference.

Finally, suppose the simulated results are found to be ambiguous or illogical. In that case, the users should return to step 1 to identify whether the lack of consideration of mindsponge components induces ambiguity. Then, the procedure is repeated accordingly. We recommend the 
user refer to the methodology of three major findings indicated above to understand better how to utilize the mindsponge mechanism and bayesvl package $[7,8,9]$. Future studies are also valuable sources for reference.

Sometimes the studied issues are relatively complex and influenced by many cognitive and contextual factors simultaneously, so the expected results cannot be acquired, even using the mindsponge*bayesvl approach. Still, we firmly believe this approach will continue to serve not only the ISR team but also other scholars for improving scientific understanding in the coming years. We acknowledge that there will be some challenges when applying such a new methodology, but foreseeable benefits are worthy for scholars to embrace the approach [18] proactively.

\section{References}

[1] Minh-Hoang Nguyen. (2021). Three most important methodological discoveries by the ISR team that power research studies in the social sciences and humanities. OSF Preprints. DOI: 10.31219/osf.io/zbepj.

[2] Quan-Hoang Vuong. (2016). Global mindset as the integration of emerging socio-cultural values through mindsponge processes: A transition economy perspective. In J. Kuada (Ed.), Global Mindsets: Exploration and Perspectives (pp. 109-126). London: Routledge.

[3] Quan-Hoang Vuong \& Nancy K. Napier. (2015). Acculturation and global mindsponge: an emerging market perspective. International Journal of Intercultural Relations, 49, 354-367.

[4] Quan-Hoang Vuong et al. (2020). Bayesian analysis for social data: A step-by-step protocol and interpretation. MethodsX, 7, 100924.

[5] Quan-Hoang Vuong et al. (2020). Improving Bayesian statistics understanding in the age of Big Data with the bayesvl R package. MethodsX, 4, 100016.

[6] Viet-Phuong La \& Quan-Hoang Vuong. (2019). bayesvl: Visually learning the graphical structure of Bayesian networks and performing MCMC with 'Stan'. The Comprehensive $\mathrm{R}$ Archive Network (CRAN). Available from: https://cran.rproject.org/web/packages/bayesvl/index.html.

[7] Minh-Hoang Nguyen et al. (2021). Alice in Suicideland: Exploring the Suicidal Ideation Mechanism through the Sense of Connectedness and Help-Seeking Behaviors. International Journal of Environmental Research and Public Health, 18(7), 3681.

[8] Quan-Hoang Vuong, Minh-Hoang Nguyen, \& Tam-Tri Le. (2021). A mindsponge-based investigation into the psycho-religious mechanism behind suicide attacks. De Gruyter / Sciendo, Warsaw, Poland (forthcoming). 
[9] Quan-Hoang Vuong, Minh-Hoang Nguyen, \& Tam-Tri Le. (2021). Home scholarly culture, book selection reason, and academic performance: Pathways to book reading interest among secondary school students. European Journal of Investigation in Health, Psychology and Education, 12(2), in press.

[10] Quan-Hoang Vuong et al. (2018). Cultural additivity: behavioural insights from the interaction of Confucianism, Buddhism and Taoism in folktales. Palgrave Communications, 4(1), 143.

[11] Quan-Hoang Vuong et al. (2020). On how religions could accidentally incite lies and violence: Folktales as a cultural transmitter. Palgrave Communications, 6(1), 82.

[12] Quan-Hoang Vuong et al. (2021). On the environment-destructive probabilistic trends: a perceptual and behavioral study on video game players. Technology in Society, 65, 101530.

[13] Quan-Hoang Vuong. (2021). The semiconducting principle of monetary and environmental values exchange. Economics and Business Letters, 10(3), 1-9.

[14] Quan-Hoang Vuong (2020). Reform retractions to make them more transparent. Nature, 582, 149.

[15] Quan-Hoang Vuong. (2018). The (ir)rational consideration of the cost of science in transition economies. Nature Human Behaviour, 2(1), 5-5.

[16] Minh-Hoang Nguyen. (2021). Bayesian inference: The enabler of Mindsponge philosophy. OSF Preprints. DOI: 10.31219/osf.io/n3jcx.

[17] Minh-Hoang Nguyen et al. (2021). On the working of the mindsponge concept and the bayesvl R package. OSF Preprints. DOI: 10.31219/osf.io/jhg7b.

[18] Quan-Hoang Vuong. (2019). Breaking barriers in publishing demands a proactive attitude. Nature Human Behaviour, 3, 1034. 